\title{
The DECAF Score: predicting hospital mortality in exacerbations of chronic obstructive pulmonary disease
}

\author{
John Steer, ${ }^{1}$ John Gibson, ${ }^{2}$ Stephen C Bourke ${ }^{1,2}$
}

\begin{abstract}
- Additional materials are published online only. To view these files please visit the journal online (http://dx.doi.org/ 10.1136/thoraxjnl-2012202103).

'Department of Respiratory Medicine, North Tyneside General Hospital, Rake Lane, North Shields, UK

${ }^{2}$ Newcastle University, Framlington Place, Newcastle-upon-Tyne, UK
\end{abstract}

\section{Correspondence to} Dr John Steer, North Tyneside General Hospital, Northumbria Health NHS Foundation Trust, Rake Lane, North Shields, Tyne and Wear, NE29 8NH, UK; john_steer@hotmail.com

Received 1 May 2012 Accepted 12 July 2012 Published Online First 15 August 2012

\begin{abstract}
Background Despite exacerbations of chronic obstructive pulmonary disease (COPD) being both common and often fatal, accurate prognostication of patients hospitalised with an exacerbation is difficult. For exacerbations complicated by pneumonia, the CURB-65 prognostic tool is frequently used but its use in this population is suboptimal.
\end{abstract}

Methods Consecutive patients hospitalised with an exacerbation of COPD were recruited. Admission clinical data and inhospital death rates were recorded. Independent predictors of outcome were identified by logistic regression analysis and incorporated into a clinical prediction tool.

Results 920 patients were recruited: mean (SD) age was 73.1 (10.0) years; $53.9 \%$ were female subjects; mean (SD) forced expiratory volume in one second was 43.6 (17.2) \% predicted; and 96 patients (10.4\%) died in hospital. The five strongest predictors of mortality (extended MRC Dyspnoea Score, eosinopenia, consolidation, acidaemia, and atrial fibrillation) were combined to form the Dyspnoea, Eosinopenia, Consolidation, Acidaemia and atrial Fibrillation (DECAF) Score. The Score, which underwent internal bootstrap validation, showed excellent discrimination for mortality (area under the receiver operator characteristic curve $=0.86,95 \% \mathrm{Cl} 0.82$ to 0.89 ) and performed more strongly than other clinical prediction tools. In the subgroup of patients with coexistent pneumonia $(n=299), D E C A F$ was a significantly stronger predictor of mortality than CURB-65.

Conclusions The DECAF Score is a simple yet effective predictor of mortality in patients hospitalised with an exacerbation of COPD and has the potential to help clinicians more accurately predict prognosis, and triage place and level of care to improve outcome in this common condition.

\section{INTRODUCTION}

In acute exacerbations of chronic obstructive pulmonary disease (AECOPD) requiring hospitalisation, inhospital mortality was $7.7 \%$ in the 2008 UK National COPD Audit. ${ }^{1}$ A robust clinical prediction tool, developed from a large prospective cohort of unselected admissions, could assist decisions regarding: location of care; early escalation of care; appropriateness for end-of-life care; and suitability for early supported hospital discharge and therefore could help to reduce morbidity and

\section{Key messages}

What is the key question?

- Can inhospital mortality in patients hospitalised with acute exacerbations of chronic obstructive pulmonary disease be predicted using a simple, robust prognostic tool?

\section{What is the bottom line?}

- A simple predictive tool (the Dyspnoea, Eosinopenia, Consolidation, Acidaemia and atrial Fibrillation Score), using indices routinely available at the time of hospital admission, can accurately predict inhospital mortality in patients hospitalised with acute exacerbations of chronic obstructive pulmonary disease.

\section{Why read on?}

- Application of the Dyspnoea, Eosinopenia, Consolidation, Acidaemia and atrial Fibrillation Score may assist clinical decision making with regard to: early supported hospital discharge; escalation of care; or early discussion of palliative care.

mortality and direct the most efficient use of resources.

In stable COPD, prognostic indices have been thoroughly investigated and tools predicting mortality risk, such as the BODE Score, ${ }^{2}$ are well established. However, prognostic research in exacerbations requiring hospitalisation has been limited, and there appears to be little common ground between predictors of mortality in stable disease and during AECOPD. ${ }^{3}$ Furthermore, none of the prognostic tools developed in stable disease have been tested on hospitalised patients, and most require clinical measurements not routinely available at hospital admission. Of the prognostic tools proposed for use in AECOPD requiring hospital admission, most were derived in highly selected, ${ }^{4-7}$ rather than unselected, ${ }^{89}$ patients.

AECOPD are often complicated by radiographic consolidation; for example, in two large UK national audits, this was reported in $16 \%$ of all admissions ${ }^{1}$ and in more than $34 \%$ of patients receiving ventilatory assistance. ${ }^{10}$ We are aware that practice varies over whether such individuals are included under the diagnosis of AECOPD, but most studies of prognosis in AECOPD requiring 
hospitalisation have not excluded patients with complicating consolidation. ${ }^{4} 58911$ Furthermore, CT scanning in AECOPD often shows consolidation not visible by plain radiography ${ }^{12} 13$ and the severity of airway obstruction and spectrum of pathogens in pneumonic and non-pneumonic exacerbations are similar. ${ }^{14}$ Pneumonic AECOPD are not simply treated as pneumonia, but require specific management of the AECOPD, including controlled oxygen therapy, corticosteroids, nebulised bronchodilators and, if respiratory acidaemia is present, noninvasive ventilation. ${ }^{15}$ We have, therefore, followed the practice of not excluding such patients.

Currently, in patients hospitalised with AECOPD complicated by consolidation, the CURB-65 (Confusion, Urea, Respiratory Rate, Blood pressure, Age > 65) community acquired pneumonia prognostic score ${ }^{16}$ is often used to risk assess and guide antibiotic therapy. ${ }^{17}$ However, we have recently shown the use of CURB-65 in patients with AECOPD and consolidation to be suboptimal, ${ }^{18}$ and a clinical prediction tool that can accurately risk stratify all patients with AECOPD is needed.

\section{METHODS}

Consecutive patients admitted to one of two neighbouring acute hospitals, representing diverse catchment areas, were recruited between December 2008 and June 2010. Approval was granted by the local National Health Service Research Ethics Committee who advised that individual patient consent was not required.

Inclusion criteria were: primary diagnosis of AECOPD supported by spirometric evidence of airflow obstruction (forced expiratory volume in one second $\left(\mathrm{FEV}_{1}\right) /$ forced vital capacity $(\mathrm{FVC})<0.70)$ when clinically stable; age $\geq 35$ years; smoking history of $\geq 10$ cigarette pack years; and admission from the primary residence. Criteria for exclusion were: previous inclusion in the study; domiciliary ventilation; comorbidity expected to limit survival to $<12$ months (principally metastatic malignancy); or a primary reason for admission other than AECOPD. Patients were managed at the discretion of their treating physicians.

\section{Data collection}

Socio-demographic and clinical data were collected on admission (online supplementary table E1). Stable-state dyspnoea was assessed using the extended Medical Research Council (MRC) dyspnoea (eMRCD) ${ }^{18}$ Score (online supplementary table E2); this subdivides patients too breathless to leave the house unaided (traditional MRCD 5) into those able independently to manage washing and/or dressing (eMRCD 5a) and those requiring assistance with both (eMRCD $5 b$ ). We have shown in the same population reported here that the inhospital death rate for eMRCD $5 \mathrm{~b}(33.1 \%)$ is substantially higher than eMRCD $5 \mathrm{a}$ $(17.3 \%)$ and that the eMRCD Score is more strongly associated with inhospital mortality than the traditional score. ${ }^{18}$ Details of comorbidity and maintenance medications were obtained from the clinical notes. The first haematological, biochemical and arterial blood gas results (and inspired concentration of oxygen, if documented) performed by the time of, or requested at, initial senior physician review (performed at least twice daily) were recorded. The presence of new consolidation on a chest radiograph at the time of a senior physician review was recorded. The presence of atrial fibrillation was confirmed by ECG at the time of hospital admission. Patients who died in hospital were identified from hospital records.

\section{Statistical methods}

For variables with $<20 \%$ missing, data were assumed to be missing at random and missing values were imputed using the expectation-maximisation algorithm (EM analysis). ${ }^{19}$ Univariate analyses were performed with and without imputed data and final conclusions were unchanged. Proportions, means with $\mathrm{SD}$ or medians with inter-quartile ranges were used to characterise the patient sample. Bivariate comparisons (table 1) were performed using Student t test (parametric data), MannWhitney $U$ test (non-parametric data) and $\chi^{2}$ test (categorical data). Multicollinearity between potential predictor variables was assessed and managed according to recommendations by Field. ${ }^{20}$

To develop a predictive tool, variables univariately associated $(p<0.10)$ with mortality were dichotomised or categorised and retained for multivariate testing. Cut-off points were identified using the following hierarchy: visual inspection of the receiver operator characteristic (ROC) curve; a clinically relevant cut-off; or a median split. Variables with $<10 \%$ of the population in one group were excluded. Eligible variables were regressed against inhospital mortality using backward stepwise methodology. Goodness-of-fit was assessed by Hosmer-Lemeshow statistic, ${ }^{21}$ and analysis of studentised residuals and leverage values identified and evaluated outliers. ${ }^{20}$

For pragmatic reasons, the five independent predictors with the largest regression coefficients were included in the final clinical prediction tool. Scores for each predictor were assigned relative to the regression coefficient. ${ }^{22}$ Performance of the tool was assessed by measuring the area under the ROC curve (AUROC). Internal validation of the tool was assessed by calculating the AUROC of 10000 bootstrapped samples. ${ }^{23}$ Two dummy prognostic models were developed using: all significant independent categorical predictors of mortality and a 'full' model containing all significant independent predictors in their original form (ie, with variables on a continuous scale where appropriate). Last, the AUROC between the two dummy prognostic models and the developed clinical prediction tool were compared. ${ }^{24}$ Statistical analyses were performed by the authors, using SPSS V.15 for Windows and SigmaPlot 11 (Systat Software Inc, San Jose, CA, USA). Exact methods were used for categorical data and a two-sided $p$ value $<0.05$ was taken as statistically significant.

\section{RESULTS}

In all, 920 patients were recruited and entered in the study. All eligible patients were included and none were lost to follow-up. Imputation of missing data by EM analysis was performed for: serum glucose (19.3\% missing); frequency of AECOPD in the previous year (15.2\%); spirometry within 2 years of admission $(14.3 \%$; of whom all had spirometry confirming airflow obstruction, performed at an earlier date); serum albumin (7.3\%); admission arterial blood gas (6.3\%); body mass index (BMI) (4.3\%); potassium (1.4\%); and respiratory rate, temperature, sodium, haemoglobin, white cell count, eosinophil count, urea and $\mathrm{C}$ reactive protein (all $<1 \%$ missing).

The mean age of the patients was 73.1 (SD 10.0) and 53.9\% were female subjects (table 1). Most patients had severe airflow obstruction (mean $\mathrm{FEV}_{1} \%$ predicted 43.6, SD 17.2); mean BMI was normal $\left(24.6 \mathrm{~kg} / \mathrm{m}^{2}\right.$, SD 6.3) although $16.7 \%$ were underweight (BMI $<18.5 \mathrm{~kg} / \mathrm{m}^{2}$ ). Approximately a third of patients had radiographic evidence of consolidation. Comparison between the patients admitted to the two study hospitals (online supplementary table E3) showed a minor difference between average $\mathrm{FEV}_{1}$ (44.8 vs $42.1 \%$ predicted) but otherwise the populations were similar.

Overall, 257 patients (27.9\%) had acidaemic respiratory failure $\left(\mathrm{pH}<7.35\right.$ and $\mathrm{p}_{\mathrm{a}} \mathrm{CO}_{2}>6 \mathrm{kPa}$ ) during their hospital stay (present at admission in 178 patients). Of these, 195 patients 
$(21.6 \%)$ received non-invasive ventilation; four required immediate intubation and ventilation; and 58 either improved with medical therapy or were not ventilated for clinical reasons. Of the 51 patients with severe dyspnoea (eMRCD $5 b$ ) who met the criteria for assisted ventilation ( $\mathrm{pH}<7.35$ and $\mathrm{p}_{\mathrm{a}} \mathrm{CO}_{2}>6 \mathrm{kPa}$ ), 44 received it and seven were not ventilated, but instead received end-of-life care.

In total, 96 (10.4\%) died during their hospital stay: 86 from respiratory disease and 10 from non-respiratory disease. Significant differences between survivors and non-survivors are shown in table 1.
Using the methods described above, the following categorical variables were entered in a backward stepwise logistic regression analysis: age $\geq 80$ years; living independently; $\geq 3$ hospital admissions in the previous year; eMRCD (groups $1-4$ combined and used as reference category); $\mathrm{FEV}_{1}<50 \%$ predicted; diastolic blood pressure $\leq 60 \mathrm{~mm} \mathrm{Hg}$; respiratory rate $\geq 30 / \mathrm{min}$; temperature $<37^{\circ} \mathrm{C}$; BMI $<18.5 \mathrm{~kg} / \mathrm{m}^{2}$; recent weight loss $\geq 5 \%$; $\mathrm{pH}<7.3$; potassium $\geq 5 \mathrm{mmol} / \mathrm{l}$; urea $\geq 7 \mathrm{mmol} / 1$; creatine $\geq 100 \mu \mathrm{mol} / \mathrm{l}$; albumin $<36 \mathrm{~g} / \mathrm{l}$; glucose $\geq 8 \mathrm{mmol} / \mathrm{l}$; haemoglobin $<12 \mathrm{~g} / \mathrm{dl}$; neutrophil count $\geq 9 \times 10^{9} / 1$; C reactive protein $\geq 50 \mathrm{mg} / \mathrm{l}$; eosinophil count $<0.05 \times 10^{9} / 1$; long-term oxygen

Table 1 Population description and comparison between survivors and those who died inhospital

\begin{tabular}{|c|c|c|c|c|}
\hline Variable & Total population, $n=920$ & Survived to discharge, $n=824$ & Died in hospital, $n=96$ & p Value* \\
\hline \multicolumn{5}{|l|}{ Socio-demographic details } \\
\hline Age (years) & $73.1(10.0)$ & $72.3(10.0)$ & $79.2(8.0)$ & $<0.001$ \\
\hline Female, \% & 53.9 & 54.2 & 51.0 & 0.59 \\
\hline $\begin{array}{l}\text { Smoking burden (cigarette pack } \\
\text { years), median (IQR) }\end{array}$ & $45(32-60)$ & $45(32-60)$ & $42.5(30-60)$ & 0.69 \\
\hline Institutional care, \% & 6.5 & 5.2 & 17.7 & $<0.001$ \\
\hline Living independently, \% & 77.1 & 79.9 & 53.1 & $<0.001$ \\
\hline Housebound, \% & 34.2 & 28.9 & 80.2 & $<0.001$ \\
\hline \multicolumn{5}{|l|}{ Markers of disease severity } \\
\hline $\begin{array}{l}\text { Number of hospital admissions in } \\
\text { previous year, median (IOR) }\end{array}$ & $0(0-1)$ & $0(0-1)$ & $1(0-2)$ & 0.096 \\
\hline $\begin{array}{l}\text { Number of AECOPD in previous year, } \\
\text { median (IQR) }\end{array}$ & $3(1-4)$ & $3(1-4)$ & $3(2-4)$ & 0.57 \\
\hline $\mathrm{FEV}_{1}(\%$ predicted $) \ddagger$ & $43.6(17.2)$ & $44.0(17.4)$ & $39.9(14.2)$ & 0.010 \\
\hline FVC (litre)‡ & $2.15(0.8)$ & $2.18(0.8)$ & $1.86(0.6)$ & $<0.001$ \\
\hline eMRCD, median (IOR) & $4(4-5 a)$ & $4(3-5 a)$ & $5 a(5 a-5 b)$ & $<0.001$ \\
\hline Long-term oxygen therapy, \% & 12.4 & 11.3 & 21.9 & 0.004 \\
\hline Previous admission requiring NIV, \% & 10.9 & 10.4 & 14.6 & 0.23 \\
\hline Cor pulmonale, \% & 10.0 & 9.8 & 11.5 & 0.72 \\
\hline Long-term prednisolone, \% & 9.1 & 8.7 & 12.5 & 0.23 \\
\hline Home nebulised therapy, \% & 16.6 & 16.6 & 16.7 & 0.99 \\
\hline \multicolumn{5}{|l|}{ Comorbidity } \\
\hline Cerebrovascular disease, \% & 14.0 & 12.6 & 26.0 & 0.001 \\
\hline Ischaemic heart disease, \% & 29.3 & 29.5 & 28.1 & 0.81 \\
\hline Hypertension, \% & 39.6 & 39.2 & 42.7 & 0.51 \\
\hline Diabetes, \% & 14.8 & 14.7 & 15.6 & 0.88 \\
\hline Atrial fibrillation, \% & 12.5 & 10.9 & 26.0 & $<0.001$ \\
\hline Left ventricular dysfunction, \% & 7.3 & 7.4 & 6.3 & 0.84 \\
\hline Chronic kidney disease, \% & 6.7 & 5.7 & 15.6 & 0.001 \\
\hline Cognitive impairment, \% & 5.4 & 4.6 & 12.5 & 0.003 \\
\hline \multicolumn{5}{|l|}{ Admission clinical data $†$} \\
\hline Purulent sputum, \% & 51.3 & 52.6 & 39.6 & 0.020 \\
\hline Ineffective cough, \% & 11.8 & 9.3 & 33.3 & $<0.001$ \\
\hline Pedal oedema, \% & 27.7 & 26.9 & 34.5 & 0.16 \\
\hline Acute confusion, \% & 12.6 & 10.0 & 35.4 & $<0.001$ \\
\hline Heart rate/min & $102.7(20.8)$ & $102.7(20.5)$ & $102.7(23.3)$ & 0.98 \\
\hline Systolic blood pressure (mm $\mathrm{Hg})$ & $139.3(28.4)$ & $139.7(28.1)$ & $135.4(30.6)$ & 0.16 \\
\hline Diastolic blood pressure $(\mathrm{mm} \mathrm{Hg})$ & $76.2(17.0)$ & $76.6(16.7)$ & $72.8(19.1)$ & 0.038 \\
\hline Respiratory rate/min & $26.0(6.3)$ & $25.8(6.1)$ & $27.8(7.6)$ & 0.004 \\
\hline Temperature $\left({ }^{\circ} \mathrm{C}\right)$, median (IOR) & $36.9(36.4-37.5)$ & $36.9(36.4-37.6)$ & $36.8(36.2-37.3)$ & 0.095 \\
\hline Oxygen saturation (\%), median (IQR) & $92(87-96)$ & $92(87-96)$ & $92(86-96)$ & 0.99 \\
\hline BMI $\left(\mathrm{kg} / \mathrm{m}^{2}\right)$ & $24.6(6.3)$ & $24.8(6.3)$ & $22.5(6.1)$ & 0.001 \\
\hline Weight loss >5\%, \% & 24.6 & 22.5 & 42.7 & $<0.001$ \\
\hline Radiographic consolidation, \% & 32.5 & 29.0 & 62.5 & $<0.001$ \\
\hline \multicolumn{5}{|l|}{ Arterial blood gas values $†$} \\
\hline $\mathrm{pH}$, median (IOR) & $7.41(7.36-7.45)$ & $7.42(7.37-7.45)$ & $7.38(7.28-7.45)$ & 0.001 \\
\hline $\mathrm{p}_{\mathrm{a}} \mathrm{O}_{2}(\mathrm{kPa})$, median (IQR) & $8.7(7.3-10.7)$ & $8.7(7.3-10.5)$ & $8.4(7.1-12.7)$ & 0.82 \\
\hline $\mathrm{p}_{\mathrm{a}} \mathrm{CO}_{2}(\mathrm{kPa})$, median (IQR) & $5.9(4.9-7.5)$ & $5.8(4.9-7.3)$ & $6.4(5.2-9.2)$ & 0.004 \\
\hline Bicarbonate $(\mathrm{mmol} / \mathrm{l})$ & $29.1(6.5)$ & $29.0(6.3)$ & $30.0(8.0)$ & 0.22 \\
\hline Acidotic exacerbation, $\% \S$ & 19.3 & 18.1 & 30.2 & 0.006 \\
\hline
\end{tabular}


Table 1 Continued

\begin{tabular}{|c|c|c|c|c|}
\hline Variable & Total population, $n=920$ & Survived to discharge, $n=824$ & Died in hospital, $n=96$ & p Value* \\
\hline \multicolumn{5}{|l|}{ Laboratory investigations $\dagger$} \\
\hline Sodium (mmol/l) & $136.3(4.6)$ & $136.3(4.5)$ & $136.7(5.0)$ & 0.39 \\
\hline Potassium (mmol/l) & $4.32(0.6)$ & $4.3(0.5)$ & $4.5(0.7)$ & 0.002 \\
\hline Urea $(\mathrm{mmol} / \mathrm{l})$, median (IQR) & $6.5(4.7-9.3)$ & $6.3(4.6-8.8)$ & $9.5(6.0-14.2)$ & $<0.001$ \\
\hline Creatine $(\mu \mathrm{mol} / \mathrm{l})$, median (IOR) & $93(77-114)$ & $92(77-112)$ & $100(75-148)$ & 0.049 \\
\hline Chloride $(\mathrm{mmol} / \mathrm{l})$ & $98.6(8.8)$ & $98.7(9.1)$ & $97.9(6.1)$ & 0.41 \\
\hline Albumin $(g / l)$ & $38.4(4.8)$ & $38.7(4.6)$ & $35.4(5.3)$ & $<0.001$ \\
\hline Glucose $(\mathrm{mmol} / \mathrm{l})$, median (IQR) & $6.9(6.0-8.1)$ & $6.9(6.0-8.0)$ & $7.4(6.0-8.9)$ & 0.030 \\
\hline Haemoglobin $(g / d)$ & $13.6(1.9)$ & $13.6(1.9)$ & $13.0(2.2)$ & 0.004 \\
\hline White cell count $\left(\times 10^{9} /\right)$, median (IQR) & $12.0(9.1-15.5)$ & $11.8(9.1-15.3)$ & $12.7(9.5-17.1)$ & 0.070 \\
\hline Neutrophil count $\left(\times 10^{9} /\right)$, median (IQR) & $9.2(6.9-12.8)$ & $9.1(6.8-12.6)$ & $10.5(7.7-15.2)$ & 0.007 \\
\hline Eosinophil count $\left(\times 10^{9} / l\right)$, median (IOR) & $0.1(0-0.2)$ & $0.1(0-0.2)$ & $0(0-0.1)$ & $<0.001$ \\
\hline CRP (mg/l), median (IOR) & $42(11-117)$ & $36(10-111)$ & $89(30-145)$ & $<0.001$ \\
\hline
\end{tabular}

All figures quote mean (SD) unless otherwise stated.

*Comparison of survivors and those who died in hospital.

†At the time of hospital admission.

$\ddagger$ Performed within 2 years of admission; self-reported unintentional weight loss during the preceding 6 months.

$\S \mathrm{pH}<7.35$ and $\mathrm{p}_{\mathrm{a}} \mathrm{CO}_{2}>6 \mathrm{kPa}$.

AECOPD, acute exacerbations of chronic obstructive pulmonary disease; BMI, body mass index; CRP, C reactive protein; eMRCD, extended MRC dyspnoea; FEV1, forced expiratory volume in one second; FVC, forced vital capacity; NIV, non-invasive ventilation.

therapy; atrial fibrillation; cerebrovascular disease; purulent sputum; acute confusion; ineffective cough; and radiographic consolidation.

The resulting regression model (table 2 ) accounted for $42 \%$ of the variance in the outcome variable (Nagelkerke $R^{2}=0.42$ ) and was a satisfactory fit to the data (Hosmer-Lemeshow statistic $=0.39$ ). No regression assumptions were violated by our model and none of the small number of statistical outliers significantly influenced the model. For comparison, the full regression model including all variables in their original form is shown in online supplementary table E4. Except for low BMI, all independent categorical predictors (table 2 ) were also significant predictors in the full model (online supplementary table E4).

In order to develop a simple and easy to use tool, the strongest five categorical variables were selected and relative weights assigned according to the regression coefficient (B). Table 3 shows calculation of the Dyspnoea, Eosinopenia, Consolidation, Acidaemia and atrial Fibrillation (DECAF) Score.

The area under the DECAF Score ROC curve for predicting inhospital mortality was 0.86 (95\% CI 0.82 to 0.89 ) (figure 1), and the bootstrapped AUROC was 0.86 (0.82 to 0.89 ), indicating

Table 2 Independent categorical predictors of inhospital mortality

\begin{tabular}{llll}
\hline Variable & B & Odds ratio (95\% Cl) & Significance \\
\hline eMRCD 1-4 & & 1 & \\
eMRCD 5a & 1.63 & $5.11(2.62$ to 9.97$)$ & $<0.001$ \\
eMRCD 5b & 1.99 & $7.30(3.77$ to 14.2$)$ & $<0.001$ \\
Coexistent consolidation & 1.06 & $2.88(1.69$ to 4.90$)$ & $<0.001$ \\
Eosinophil count & 1.02 & $2.76(1.58$ to 4.83$)$ & 0.001 \\
$<0.05 \times 10^{9} / \mathrm{l}$ & & & \\
$\mathrm{pH}<7.3$ & 0.99 & $2.68(1.41$ to 5.09$)$ & 0.003 \\
$\mathrm{AF}$ & 0.98 & $2.66(1.39$ to 5.09$)$ & 0.003 \\
Ineffective cough & 0.94 & $2.57(1.37$ to 4.84$)$ & 0.003 \\
Albumin $<36 \mathrm{~g} / \mathrm{l}$ & 0.84 & $2.32(1.36$ to 3.96$)$ & 0.002 \\
Cerebrovascular disease & 0.70 & $2.02(1.18$ to 3.42$)$ & 0.037 \\
Age $\geq 80$ & 0.70 & $2.01(1.18$ to 3.42$)$ & 0.011 \\
BMl $<18.5 \mathrm{~kg} / \mathrm{m}^{2}$ & 0.60 & $1.83(1.00$ to 3.33$)$ & 0.049 \\
Intercept & -4.30 & & \\
\hline
\end{tabular}

$\mathrm{AF}$, atrial fibrillation; $\mathrm{BMI}$, body mass index; eMRCD, extended MRC dyspnoea. good internal validity. Compared with the DECAF Score, there were small but statistically significant differences between (a) the AUROC for a dummy categorical tool (using all the variables listed in table 2) (0.89 vs $0.86, p=0.038$ ) and (b) the AUROC for the full regression model using all variables in their original form (online supplementary table E4) (0.90 vs $0.86, \mathrm{p}<0.001$ ). The DECAF Score performed strongly in each participating hospital (AUROC $=0.88$ vs $0.84, p=0.27$ ) and if the population was stratified according to $\mathrm{FEV}_{1}$ (AUROC $\mathrm{FEV} 1<50 \%=0.857$ vs $\left.\mathrm{AUROC}_{\mathrm{FEV} 1} \geq 50 \%=0.850, \mathrm{p}=0.91\right)$. Inhospital and 30-day death rates, and sensitivity and specificity for inhospital mortality, for the DECAF Score are shown in table 4.

In our cohort, the DECAF Score performed significantly better for the prediction of inhospital mortality than: the Acute Physiology and Chronic Health Evaluation (APACHE) II prognostic index ${ }^{25}$ (AUROC $=0.73$, DECAF vs APACHE II $p<0.001$ ); the COPD and Asthma Physiology Score ${ }^{5}$ (AUROC $=0.71$, $\mathrm{p}<0.001$ ); and the BAP-65 (elevated Blood urea nitrogen, Altered mental status, Pulse $>109 / \mathrm{min}$, Age $>65$ years) Score ${ }^{8}$ (AUROC $=0.68, \mathrm{p}<0.001$ ) which have all been proposed as useful predictive instruments in AECOPD (figure 1). ${ }^{4} 526$

DECAF was a significantly stronger predictor of inhospital mortality than CURB-65 for both patients with (AUROC $=0.77$ vs $0.66, p=0.003, n=299$ ) (figure 2, panel $A$ ) and without (AUROC $=0.87$ vs $0.72, \mathrm{p}=0.002, \mathrm{n}=621$ ) (figure 2 , panel $\mathrm{B}$ ) consolidation. As CURB-65 was introduced to predict 30-day

Table 3 The DECAF Score

\begin{tabular}{lc}
\hline Variable & Score \\
\hline Dyspnoea & \\
eMRCD 5a & 1 \\
eMRCD 5b & 2 \\
Eosinopenia $\left(<0.05 \times 10^{9} / I\right)$ & 1 \\
Consolidation & 1 \\
Acidaemia (pH $<7.3)$ & 1 \\
Atrial fibrillation & 1 \\
Total DECAF Score & 6 \\
\hline DECAF, Dyspnoea, Eosinopenia, Consolidation, Acidaemia and atrial \\
Fibrillation; eMRCD, extended MRC dyspnoea.
\end{tabular}




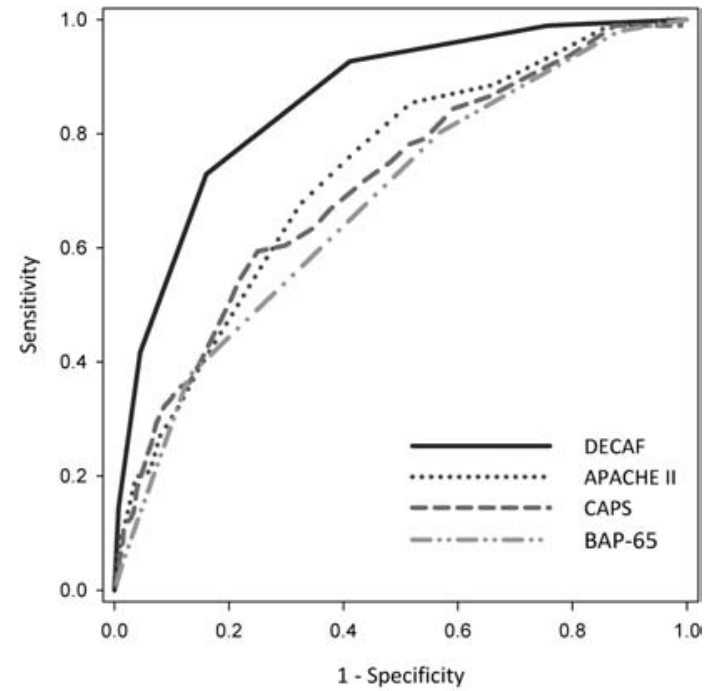

APACHE II, Acute Physiology and Chronic Health Evaluation II; BAP-65 Blood urea nitrogen, Altered mental status, Pulse $>109 / \mathrm{min}$, Age $>65$ years; CAPS, COPD and Asthma Physiology Score; COPD,chronic obstructive pulmonary disease.

Figure 1 Receiver operator characteristic curve showing discrimination of Dyspnoea, Eosinopenia, Consolidation, Acidaemia and atrial Fibrillation (DECAF) Score for inhospital mortality in the total population.

mortality, we also assessed the use of DECAF for predicting 30-day mortality. The AUROC of DECAF for the prediction of 30-day mortality was 0.82 (0.78 to 0.86 ) and, in the subgroup with consolidation, it was a stronger predictor than CURB-65 (AUROC $=0.75$ vs $0.64, \mathrm{p}=0.003$ ).

\section{DISCUSSION}

In patients hospitalised with AECOPD, a simple prognostic tool, incorporating clinical and laboratory information available routinely on admission, accurately predicted inhospital mortality, and performed more strongly than other proposed prognostic scores. In the subgroup of patients with consolidation complicating AECOPD, the DECAF Score was a stronger predictor of mortality than CURB-65.

Our study conclusions are strengthened by the prospective recruitment of a large number of sequential patients; in particular, the two institutions reflect different catchment areas (urban and rural) with different structures of care and a wide range of socioeconomic status represented and our inclusion criteria ensured a broad spectrum of patients with AECOPD were recruited. Furthermore, performance of the tool on internal validation was strong and, although external validation is necessary, our findings support the generalisability of the conclusions. Allowing for the difference in proportions with consolidation in our study $(32.5 \%)$ and the UK national audit (16\%), the death (non-pneumonic $\mathrm{AECOPD}=5.8 \%$, pneumonic $\mathrm{AECOPD}=20.1 \%$ ) and readmission rates of our patients were in line with UK national data. Practice regarding the inclusion of patients with pneumonic AECOPD under the diagnosis of AECOPD does, however, vary between hospitals and, therefore, we suggest that in future national audits, mortality data should be stratified by the presence or absence of complicating consolidation.

We are aware that comparisons between other prognostic tools and the DECAF Score in its derivation cohort may introduce bias in favour of the DECAF tool. However, the large number of patients included, the generalisability of our methodology and the size of the differences in prognostic performance suggest that the stronger performance of DECAF compared with the other tools assessed is likely to be valid.

We acknowledge certain limitations in the way the data were obtained, but the study was designed to reflect the 'real life' clinical situation. Clinical information was gathered by medical, nursing and research staff using standard protocols, and the presence or absence of consolidation was recorded by the admitting medical team. To ensure generalisability, we included all eligible patients and did not interfere with management provided by the attending physicians. We recognise that severe disability is likely to have been an important consideration in determining the management of individual patients. However, our findings do not appear to be explained by early introduction of palliative care, or limiting the level of care, in this population because, even among patients with the most severe limitation (eMRCD 5b), most of those potentially eligible for assisted ventilation received it. Although missing data were relatively few, data had to be imputed for a small number of variables. To ensure that imputation using EM analysis did not bias our results, univariate analyses were repeated using the original dataset and the conclusions were unchanged.

Compared with the DECAF Score, there were small but statistically significant improvements in discrimination when comparing dummy prognostic tools based on either all independent categorical predictors (table 2) or all independent predictors in their original form (online supplementary table E4). However, we consider that these minor improvements are outweighed by the ease with which the simpler DECAF tool can be clinically applied. Roche et $a l^{9}$ derived a predictive tool from 794 patients attending an emergency department with AECOPD. Their prognostic score showed good discrimination for inhospital mortality (AUROC $=0.79$ ) but included subjectively assessed signs of clinical severity. The DECAF Score performed more strongly in our population than the tool described by Roche et al in its derivation cohort and, furthermore, the prognostic indices included in the DECAF Score are objective with little potential for variable interpretation.

Table 4 DECAF Score and inhospital mortality

\begin{tabular}{llllll}
\hline DECAF Score & $\mathbf{n}$ & Inhospital mortality, $\%$ & Sensitivity $^{*}$ & Specificity $^{*}$ & 30-day mortality, \% \\
\hline 0 & 201 & 0.5 & 1 & 0 & 1.5 \\
1 & 291 & 2.1 & 0.99 & 0.24 & 3.8 \\
2 & 226 & 8.4 & 0.93 & 0.59 & 11.9 \\
3 & 125 & 24 & 0.73 & 0.84 & 27.2 \\
4 & 57 & 45.6 & 0.42 & 0.96 & 45.6 \\
5 & 20 & 70 & 0.15 & 0.99 & 70 \\
6 & 0 & NA & NA & NA & NA
\end{tabular}

${ }^{*}$ For inhospital mortality: positive test result $=$ score $\geq$ corresponding DECAF Score; DECAF 0-1 = Low risk; $2=$ Intermediate risk; $\geq 3=$ High risk.

DECAF, Dyspnoea, Eosinopenia, Consolidation, Acidaemia and atrial Fibrillation. 
A

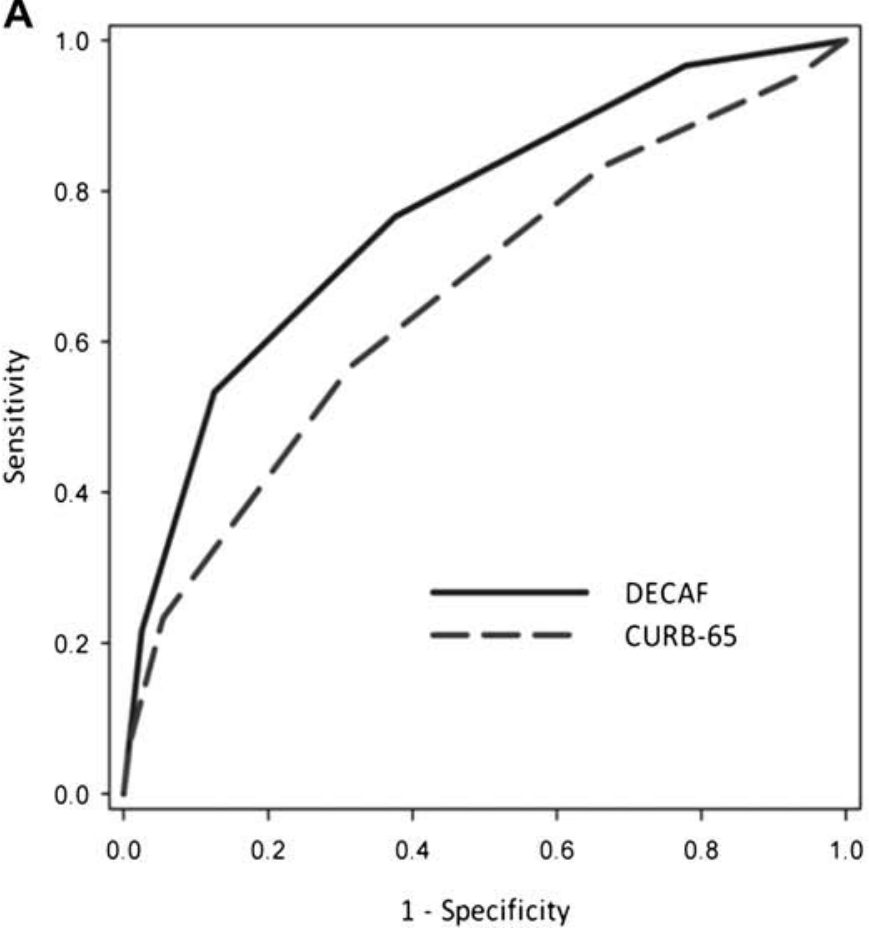

B

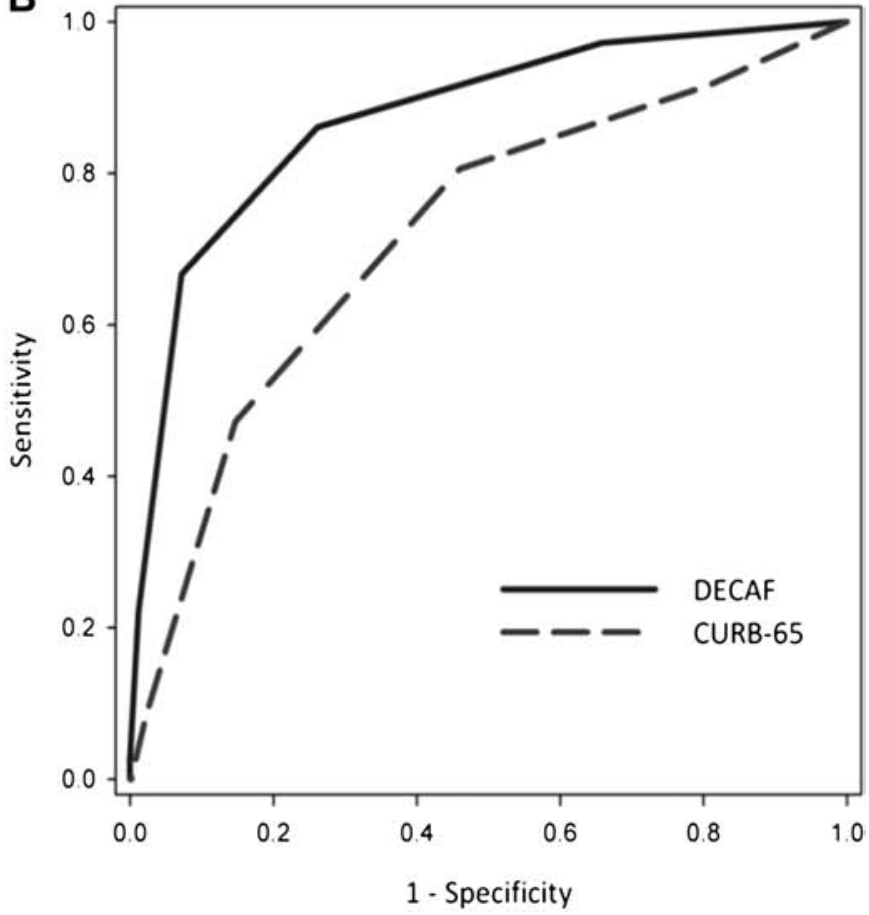

Figure 2 Receiver operator characteristic curve showing discrimination of Dyspnoea, Eosinopenia, Consolidation, Acidaemia and atrial Fibrillation (DECAF) Score and CURB-65 for inhospital mortality for patients with $(n=299$, panel A) and without $(n=621$, panel B) consolidation.

Many of the indices independently associated with higher mortality in table 2 are consistent with previously published research in AECOPD: increasing age $;^{9}{ }^{27}$ dyspnoea severity; ${ }^{9}$ low $\mathrm{BMI} ;{ }^{11}$ low $\mathrm{pH} ;{ }^{11}{ }^{28}$ long-term oxygen therapy prescription; ${ }^{28}$ cough effectiveness; ${ }^{29}$ and coexistent consolidation. ${ }^{14}$ Both cardiovascular and non-cardiovascular chronic comorbidities have been associated with higher inhospital mortality in $\mathrm{AECOPD},{ }^{30}$ but, to our knowledge, this is the first study to report in an unselected population of $\mathrm{AECOPD}$ requiring hospitalisation that both atrial fibrillation and cerebrovascular disease are independently predictive of mortality. Holland et al ${ }^{31}$ reported that eosinopenia $\left(<0.04 \times 10^{9} / 1\right)$ was associated with a higher inhospital mortality in AECOPD, but the study population was small $(n=65)$ and the role of confounders was not evaluated. Our results show that eosinopenia is a strong independent predictor of inhospital mortality. Of note, this finding is not due to a better prognosis among patients with eosinophilia, as patients with confirmed or suspected asthma were excluded, and only a small proportion had an elevated eosinophil count $\left(>0.4 \times 10^{9} / 1, \mathrm{n}=55\right)$ at admission; if the latter are excluded from analysis, our conclusions remain unchanged (results not shown). It is also noteworthy that Holland et al ${ }^{31}$ excluded individuals who had recently received oral corticosteroids, and in our study, there was no significant difference in eosinophil count between patients receiving either long-term inhaled $(p=0.38)$ or oral $(p=0.51)$ corticosteroids and those not in receipt of these therapies. Furthermore, it has previously been shown in an animal model that eosinopenia accompanies the response to acute infection and inflammation, ${ }^{32}$ independent of adrenal glucocorticosteroids, ${ }^{33}$ and may be a useful marker of sepsis in patients who are receiving intensive care. ${ }^{34} 35$ In AECOPD, the strong prognostic influence of eosinopenia may reflect the severity of the accompanying acute inflammatory response.

In community acquired pneumonia, the CURB-65 prognostic too ${ }^{16}$ effectively predicts mortality within 30 days of admission.
However, although complicating consolidation is common in AECOPD, and CURB-65 is often used in this situation, we have recently shown, in the population reported here, that its performance is suboptimal. ${ }^{18}$ Compared with CURB-65, the DECAF Score was a stronger predictor of both inhospital and 30-day mortality, and in pneumonic AECOPD, CURB-65 had only moderate performance.

The DECAF Score shows promise for the risk stratification of patients hospitalised with AECOPD. ROC analysis suggests that it has at least 'good' performance and is a stronger prognostic score than the CURB-65, APACHE or COPD and Asthma Physiology Score predictive tools. The death rates for each grade of the DECAF Score (table 4) suggest the following risk categories: DECAF 0-1 ('low risk'; inhospital mortality $=1.4 \%$ ); DECAF 2 ('moderate risk'; mortality $=8.4 \%$ ); and DECAF 3-6 ('high risk'; mortality $=34.6 \%$ ). Our findings suggest that more than half of the patients hospitalised with AECOPD can be classified as low risk of both inhospital and 30-day mortality and might therefore potentially be suitable for early supported discharge (ESD) schemes. Use of the DECAF Score might, therefore, increase access to ESD schemes as current evidence ${ }^{36}$ suggests approximately $25 \%$ of patients hospitalised with AECOPD are deemed eligible for ESD. There is no evidence that this finding reflects a low threshold for admission in our population as, compared with the 2008 UK National COPD Audit, ${ }^{1}$ a higher proportion of our patients required ventilation and the overall mortality, after correction for the relative proportions with consolidation, was similar in the two studies. On the other hand, a high DECAF Score might be used as a guide to early escalation of care, or, where appropriate, to early discussion of end-of-life care.

In conclusion, our results suggest that a simple clinical prediction tool, incorporating indices routinely available at the time of hospital admission, can accurately stratify patients hospitalised with AECOPD into clinically relevant risk 
categories and could therefore assist clinicians managing this frequently fatal condition.

Acknowledgements The authors acknowledge the following for their help with the study: Dr Nick Steen, biostatistician, Newcastle University; the respiratory and acute care physicians at the North Tyneside General Hospital and Wansbeck General Hospital; Dr Gbenga Afolabi, Respiratory Physician; Ms Elizabeth Norman and the Respiratory Specialist Nursing Team, North Tyneside General Hospital; the North Tyneside General Hospital Teaching and Research Fellow programme; Breathe North; and the National Institute of Health Research, through the Comprehensive Local Research Network.

Contributors SCB designed and obtained funding for the study. JS performed the literature search and collected the data. Data analysis and interpretation were performed by JS and supervised by SCB and JG. The manuscript was drafted by JS; SCB and JG helped with its revision.

Funding The Breathe North Appeal and the Northumbria Healthcare NHS Foundation Trust Teaching and Research Fellow Programme.

Competing interests None.

Ethics approval Ethics approval was provided by the County Durham and Tees Valley NHS Research Ethics Committee II.

Provenance and peer review Not commissioned; externally peer reviewed.

\section{REFERENCES}

1. Royal College of Physicians, British Thoracic Society, British Lung Foundation. Report of the National Chronic Obstructive Pulmonary Disease Audit 2008: Clinical Audit of COPD Exacerbations Admitted to Acute NHS Trusts across the UK. London: Royal College of Physicians, 2008.

2. Celli BR, Cote CG, Marin JM, et al. The body-mass index, airflow obstruction, dyspnea, and exercise capacity index in chronic obstructive pulmonary disease. N Engl J Med 2004;350:1005-12.

3. Steer J, Gibson GJ, Bourke SC. Predicting outcomes following hospitalization for acute exacerbations of COPD. OJM 2010;103:817-29.

4. Confalonieri M, Garuti G, Cattaruzza MS, et al. A chart of failure risk for noninvasive ventilation in patients with COPD exacerbation. Eur Respir J 2005;25:348-55.

5. Wildman MJ, Harrison DA, Welch CA, et al. A new measure of acute physiological derangement for patients with exacerbations of obstructive airways disease: the COPD and Asthma Physiology Score. Respir Med 2007;101:1994-2002.

6. Ruiz-Gonzalez A, Lacasta D, Ibarz M, et al. C-reactive protein and other predictors of poor outcome in patients hospitalized with exacerbations of chronic obstructive pulmonary disease. Respirology 2008;13:1028-33.

7. Anton A, Guell R, Gomez J, et al. Predicting the result of noninvasive ventilation in severe acute exacerbations of patients with chronic airflow limitation. Chest 2000;117:828-33

8. Tabak YP, Sun X, Johannes RS, et al. Mortality and need for mechanical ventilation in acute exacerbations of chronic obstructive pulmonary disease: development and validation of a simple risk score. Arch Intern Med 2009;169:1595-602.

9. Roche N, Zureik M, Soussan D, et al. Predictors of outcomes in COPD exacerbation cases presenting to the emergency department. Eur Respir $\mathrm{J}$ 2008:32:953-61.

10. Davidson C. 2010 Adult Non-Invasive Ventilation Audit Summary Report. The British Thoracic Society, 2011.

11. de la Iglesia F, Diaz JL, Pita S, et al. Peak expiratory flow rate as predictor of inpatient death in patients with chronic obstructive pulmonary disease. South Med J 2005;98:266-72.

12. Syrjala H, Broas M, Suramo I, et al. High-resolution computed tomography for the diagnosis of community-acquired pneumonia. Clin Infect Dis 1998;27:358-63.
13. Hagaman JT, Rouan GW, Shipley RT, et al. Admission chest radiograph lacks sensitivity in the diagnosis of community-acquired pneumonia. Am J Med Sci 2009; 337:236-40.

14. Lieberman D, Lieberman D, Gelfer $Y$, et al. Pneumonic vs nonpneumonic acute exacerbations of COPD. Chest 2002;122:1264-70.

15. Confalonieri M, Potena A, Carbone G, et al. Acute respiratory failure in patients with severe community-acquired pneumonia. A prospective randomized evaluation of noninvasive ventilation. Am J Respir Crit Care Med 1999;160:1585-91.

16. Lim WS, van der Eerden MM, Laing $R$, et al. Defining community acquired pneumonia severity on presentation to hospital: an international derivation and validation study. Thorax 2003;58:377-82.

17. Lim WS, Baudouin SV, George RC, et al. BTS guidelines for the management of community acquired pneumonia in adults: update 2009. Thorax 2009;64(Suppl 3): iii1-55.

18. Steer J, Norman EM, Afolabi OA, et al. Dyspnoea severity and pneumonia as predictors of in-hospital mortality and early readmission in acute exacerbations of COPD. Thorax 2012:67:117-21.

19. Enders CK. A Primer on Maximum Likelihood algorithms available for use with missing data. Structural Equation Modelling 2001;8:128-41.

20. Field A. Discovering Statistics Using SPSS. 3rd edn. London: SAGE, 2009.

21. Hosmer DW Jr, Lemeshow S. Goodness-of-fit tests for the multiple logistic regression model. Comm Stat 1980;A9:1043-69.

22. Sullivan LM, Massaro JM, D'Agostino RB, et al. Presentation of multivariate data for clinical use: the Framingham Study risk score functions. Stat Med 2004;23:1631-60.

23. Garcia-Granero M. Bootstrapping ROC AUC. SPSSX Discussion. University of Georgia, 2008. http://spssx-discussion.1045642.n5.nabble.com/Re-BootstrappingROC-AUC-tt1083963.html (accessed 20 Apr 2011)

24. DeLong ER, DeLong DM, Clarke-Pearson DL. Comparing the areas under two or more correlated receiver operating characteristic curves: a nonparametric approach. Biometrics 1988:44:837-45.

25. Knaus WA, Draper EA, Wagner DP, et al. APACHE II: a severity of disease classification system. Crit Care Med 1985;13:818-29.

26. Shorr AF, Sun X, Johannes RS, et al. Validation of a novel risk score for severity of illness in acute exacerbations of COPD. Chest 2011;140:1177-83.

27. Patil SP, Krishnan JA, Lechtzin N, et al. In-hospital mortality following acute exacerbations of chronic obstructive pulmonary disease. Arch Intern Med 2003;163:1180-6.

28. Bustamante-Fermosel A, De Miguel-Yanes JM, Duffort-Falco M, et al. Mortalityrelated factors after hospitalization for acute exacerbation of chronic obstructive pulmonary disease: the burden of clinical features. Am J Emerg Med 2007;25:515-22

29. Levy M, Tanios MA, Nelson D, et al. Outcomes of patients with do-not-intubate orders treated with noninvasive ventilation. Crit Care Med 2004;32:2002-7.

30. Scala R, Bartolucci S, Naldi M, et al. Co-morbidity and acute decompensations of COPD requiring non-invasive positive-pressure ventilation. Intensive Care Med 2004;30:1747-54.

31. Holland M, Alkhalil M, Chandromouli S, et al. Eosinopenia as a marker of mortality and length of stay in patients admitted with exacerbations of chronic obstructive pulmonary disease. Respirology 2010;15:165-7.

32. Bass DA, Gonwa TA, Szejda P, et al. Eosinopenia of acute infection: production of eosinopenia by chemotactic factors of acute inflammation. J Clin Invest 1980;65:1265-71.

33. Bass DA. Behaviour of eosinophil leukocytes in acute inflammation. J Clin Invest 1975; 55:1229-36.

34. Abidi K, Khoudri I, Belayachi J, et al. Eosinopenia is a reliable marker of sepsis on admission to medical intensive care units. Crit Care 2008:12:R59.

35. Shaaban H, Daniel S, Sison R, et al. Eosinopenia: is it a good marker of sepsis in comparison to procalcitonin and C-reactive protein levels for patients admitted to a critical care unit in an urban hospital? J Crit Care 2010;25:570-5.

36. Ram FS, Wedzicha JA, Wright J, et al. Hospital at home for patients with acute exacerbations of chronic obstructive pumonary disease: systematic eview of evidence. BMJ 2004;329:315. 